\title{
Epidemiological transition in Brazil, obesity and state of the art of the methods of treatment and multidisciplinary interventions of obesity
}

Keywords: public health service, multidisciplinary obesity treatment, bariatric Surgery

\section{Opinion}

Although during the last decades there has been a decrease in the statistics regarding the mortality numbers, there are some current researches indicating an increase in the morbidity numbers among the population. From the actual mortalities occurred, about 70 to $80 \%$ are closely related to obesity diseases and its comorbidities.

The Brazilian Health Ministry, knowledgeable of the current scenario and following the international orientations regarding the prevention of this pandemic, recently released two national ordinances that give a better dimension of the importance of this disease. Named the ordinances 424 and 425, they emphasize the importance of invest at the obesity prevention and treatment, besides of the enlargement of the bariatric surgeries to the people diagnosed with obesity, although the document highlight the priority is to treat this disease through the multidisciplinary treatment, based on the behavior change and reduction of the bad daily habits, that can compromise the individual's health, as sedentary lifestyle, bad food intake, among others.

At practice, through the specific literature, is clear the increase of the bariatric surgery year after year in the country, and the multidisciplinary treatments based on behavior changes keep restrict to some researches groups spread in Brazil. Therefore, the current doctoral thesis aimed to verify the epidemiological transition in Brazil, the obesity, and the state of art of the ways of treatment and multidisciplinary interventions of the obesity. This way, the study was divided in 3 distinct moments, being the first study based on the documental descriptive methodology in which aimed to analyze the Brazilian epidemiologic situation related to obesity, comorbidities, and health public politics. As finds of this study, it was verified a growing investment of the health services in Brazil, however this action is not being converted in improvements in the health of the population when observed the high prevalence of death related to the noncommunicable chronic diseases, as like the constant evolution of the weight excess in Brazil and through the high demand of the public health care system in relation of hospitalizations, medicines, consultations and medical exams. The second study, defined as a systematic review about the bariatric surgery in Brazil as a politic of obesity treatment.

The results indicated that although this method is only for the most critical cases, the service was enlarged in 2013 and in therefore, the number of people choosing this surgical procedure is rapidly increasing. The service presents positive effects in short and medium term, however, concerning the long-term effects there are some evidences of weight regain and a loss of the enhancements previously

Volume 8 Issue 3 - 2018

\author{
Anselmo Alexandre Mendes, Suzana Vieira, \\ Jane Maria Remor , Braulio Henrique \\ Magnani Branco,Carlos Andres Lopera \\ Barrero, Nelson Nardo Junior \\ Department of Physical Education, University State of Maringá, \\ Brazil
}

Correspondence: Anselmo Alexandre Mendes, Department of Physical Education, University State of Maringá, Brazil, Email profanselmol@gmail.com

Received: December 27, 2017| Published: May 15, 2018

achieved. In the third article, it has been studied by systematic review, the multidisciplinary treatments in Brazil. The results showed these programs are not offered to the population as a health service, being restricted to some research groups spread in Brazil. Considering that, a small amount of people has access to this option of treatment.

The main results of the studies were in anthropometric aspects (BMI, waist circumference, hip cinferência, increased lean body mass, decreased fat mass); psychological (improvement of emotional and psychosocial domain, satisfaction with body image and quality of life); biochemical (decrease in total cholesterol, triglycerides, insulin, HOMA-IR, increased HDL and adiponectin, reduced glucose, LDL, leptin and ghrelin); nutritional (decrease in intake of macronutrients, lipids, sugars, sodium, kcal); Hemodynamic and physical fitness (improvements in aerobic fitness, $\mathrm{VO}_{2} \max$, physical, SBP, DBP, cardíacarep frequency, strength / resistance, variability of heart frequeência). In conclusion, the design of the health programs and the investments from the national politics are not having a considering influencing regarding the improvements of the epidemiologic situation in Brazil. There must have a reformulation of the action plan, to invest on the obesity prevention and its comorbidities, to decrease this way, the number of people that choose the bariatric surgery over the multidisciplinary program of obesity treatment with focus on behavioral change, in which, it is suggested that become a permanent service of the public health care system, not only just to the treatment but also to prevent the obesity.

\section{Acknowledgements}

NEMO - group of multiprofessional obesity studies

\section{Conflicts of interest}

The authors declare there is no conflict of interest 